Horizons philosophiques

\title{
L'hypothèse de Whorf s'applique-t-elle à la philosophie? Brève réflexion sur les heurs et malheurs du rapport de la langue à la culture avec la philosophie comme toile de fond
}

\section{Roch Duval}

Volume 12, numéro 1, automne 2001

Langue : identité plurielle

URI : https://id.erudit.org/iderudit/801194ar

DOI : https://doi.org/10.7202/801194ar

Aller au sommaire du numéro

Éditeur(s)

Collège Édouard-Montpetit

ISSN

1181-9227 (imprimé)

1920-2954 (numérique)

Découvrir la revue

Citer cet article

Duval, R. (2001). L'hypothèse de Whorf s'applique-t-elle à la philosophie? Brève réflexion sur les heurs et malheurs du rapport de la langue à la culture avec la philosophie comme toile de fond. Horizons philosophiques, 12(1), 28-52.

https://doi.org/10.7202/801194ar 


\title{
L'hypothèse de Whorf s'applique-t-elle à la philosophie? Brève réflexion sur les heurs et malheurs du rapport de la langue à la culture avec la philosophie comme toile de fond*
}

\author{
Nederst substantiverne : Jorden vi står på, småting \\ der ligger og flyder, og vejene, husene. I midten ver- \\ berne: vi går, løber, handler: Øverst en himmel af \\ adjektiver: blå, truende, rolig, lysere. \\ Tomas Thøiner. 1
}

\section{Introduction}

Après une période, brève mais intense, d'immense popularité auprès de l'intelligentsia internationale - œuvrant dans des domaines aussi variés et hétérogènes tels que l'anthropologie, la psychologie, la linguistique, l'ethnologie, l'ethnolinguistique, la philosophie, pour ne nommer que ceux-là - la dénommée hypothèse de la relativité linguistique (également connue sous le vocable "hypothèse Sapir-Whorf") ${ }^{2}$ a vu le lustre la nim-

* Modeste contribution visant à commémorer le $60^{\circ}$ anniversaire du décès de Benjamin Lee Whorf (1897-1941).

1. «Au niveau inférieur les substantifs : la terre sur laquelle nous nous tenons, les petits objets qui traînent, puis les routes, les maisons. Au niveau intermédiaire les verbes : nous marchons, courons, agissons. Au niveau supérieur un ciel d'adjectifs : bleu, menaçant, serein, brillant". Strophe extraite du poème "Stase" (Stase) du poète danois Tomas Thøfner (né en 1969) reproduit dans son plus récent recueil intitulé Det synkrone, Copenhague, Borgen, 2000. (L'ensemble des traductions, sauf indication contraire, sont les miennes.)

2. Selon E.F.K. Koerner il faudrait attribuer à Harry Hoijer l'introduction du vocable «SapirWhorf Hypothesis" pour désigner la variante américaine de la relativité linguistique. La dénomination serait cependant devenue usuelle deux années plus tard alors que celle-ci apparaît dans l'introduction rédigée par John B. Carroll au volume regroupant les œuvres choisies de Whorf. Voir Harry Hoijer "The Sapir-Whorf Hypothesis" dans $\mathrm{H}$. Hoijer (ed.) Language in Culture : Proceedings of a Conference on the Interrelations of Language and Others Aspects of Culture, Chicago, Chicago University Press, 1954, p. 92-105; Language, Thought and Reality : Selected Writings of Benjamin Lee Whorf, John B. Carroll (ed.) Cambridge, Mass : MIT Press, 1956; E.F.K. Koerner, "Towards a "Full Pedigree" of the "Sapir-Whorf Hypothesis". From Locke to Lucy" dans Martin Pütz et Marjolijn H. Verspoor (ed.) Explorations in Linguistic Relativity, Amsterdam Studies in the Theory and History of Linguistic Science. Series IV Current Issues in Linguistic Theory, Volume 199, Amsterdam/Philadelphie, John Benjamins, 2000, p. 1-23. 
L'hypothèse de Whorf s'applique-t-elle à la philosophie?

Brève réflexion sur les heurs et malheurs du rapport de la langue à la culture

avec la philosophie comme toile de fond

L'histoire de la relativité linguistique remonte à des temps immémoriaux. Voir I.M. Schlesinger, "The wax and wane of Whorfian views", dans Robert $L$. Cooper et Bernard Spolsky (ed.), The Influence of Language on Culture and Thought. Essays in Honor of Joshua A. Fishman's Sixty-Fifth Birthday. Berlin-New York, Mouton de Gruyter, 1991, p. 7-44.

II me semble tout de même important de mentionner les temps forts jalonnant ce parcours : les philosophes allemands Johann Gottfried Herder (1744-1803) et (principalement) Wilhelm von Humboldt en sont les sommets. La mention de ces deux illustres tenants de la thèse de la relativité linguistique m'apparaît particulièrement importante car il arrive à l'occasion de trouver ces noms accolés à ceux de Sapir et Whorf. D'aucuns parlent même de l'hypothèse "Herder-Humboldt-Sapir-Whorf", voire même de l'hypothèse "Vico-Herder-Humboldt-Korzybski-Sapir-Whorf". Voir Robert P. Pula, "The Nietzsche-Korzybski-Sapir-Whorf Hypothesis", Etc. : A Review of General Semantics 49, (1992), p. 81-102. On pourrait même inclure dans ce groupe des supporters moins connus tels que Jost Trier, Leo Wiesgerber, le Polonais Jan Baudoin de Courtenay (1845-1929) ainsi qu'une légion d'intellectuels provenant de tous les horizons linguistiques. Le dénominateur commun à ces différentes approches se réduit à lidée de la langue (maternelle) comme image du monde (Weltansicht) ou - comme chez Humboldt - comme conception du monde (Weltanschauung). Voir chez les Polonais, A. MaÒczyk, Wspólnota jízykowa i jej obraz úwiata. Krytyczne uwagi do teorii jízykowej Leo Weisgerbera. (Le langage commun et son image du monde. Observations critiques sur la théorie linguistique de Leo Weisgerber), Zielona Góra, 1982; R. Grzegorczykowa, "Pojície jízykowego obrazu úwiata", (L'idée de l'image linguistique du monde) dans J. BartmiÒski (ed.), Jízykowy obraz úwiata. (L'image linguistique du monde), Lublin, 1990, p. 41-9 et J. Anusiewicz, «Problematyka jízykowego obrazu úwiata w pogladach niektórych jízykoznawców i filozofów niemieckich XX wieku» (Problématique de l'image linguistique du monde dans les conceptions de quelques linguistiques et philosophes allemands du XXe siècle) dans J. BartmiÒski, ibid., 1990. 287-94); Pawe $\geq$ Bytniewski, "Hipoteza Sapira-Whorfa, czily jízyk jako medium poznania" (L'hypothèse Sapir-Whorf, ou la langue comme moyen de connaissance) dans Zbys $\_a w$ MuszyÒski (ed.) J́zyk, znaczenie, rozumienie $i$ relatywizm. (Langue, signification, compréhension et relativisme), Lublin, Wyd, UMCS, p. 5-30. En ce qui concerne les théoriciens hongrois, voir Imre Farkas, "Nyelvi hatások világképünkre - a Sapir-Whorf hipotézis" (Les effets de la langue sur notre image du monde. L'hypothèse Sapir-Whorf), Kortárs No 7-8, (1964), p. 1651-5; József Hegedüs, “Nyelvtan - tudat - kreativitás» (Grammaire, connaissance, créativité) FelsöoktSzle. (février 1985), p. 86-92.

David Fielding, dans un texte intéressant, s'évertue à distancier la théorie de Sapir de celle de son dauphin et conteste du même coup la justesse de l'appellation «Hypothèse Sapir-Whorf". Voir David Fielding, "L'hypothèse Sapir", La Petite Revue de Philosophie, 7. (1986), p. 17-46. La même attitude déconstructiviste (ou critico-analytique) se retrouve chez John A. Lucy, Language diversity and thought. A reformulation of the linguistic relativity hypothesis, Studies in the Social and Cultural Foundations of Language № 12, Cambridge, Cambridge University Press, 1992, p. 17-24. Voir également Marion V. Smith, "Linguistic Relativity : On Hypothesis and Confusions", Communication \& Cognition Vol. 29, №. 1, (1996), p. 65-90. De là découle la nécessité impérieuse de cerner la teneur précise de l'énonciation de l'hypothèse de la relativité linguistique de Whorf afin de différencier celle-ci de ses proches parentes directes (telles que la position néohumboldtienne de Helmut Gipper ou bien celle de Jürgen Trabant) ou encore de la "kyrielle" des théories affines. Voir Helmut Gipper, Gibt es ein sprachliches Relativitätsprinzip? Untersuchung zur Sapir-Whorf-Hypothese, Francfort: S. Fischer, 1972; Jürgen Trabant, Traditionen Humboldts, Francfort sur le Main, Suhrkamp, 1990; 
bant d'une auréole de prestige se décatir graduellement. D'hypothèse estimée et admirée qu'elle était au début des années cinquante, elle représentait encore il n'y a pas si longtemps ${ }^{3}$ un exemple infamant de "fausse science" 4 et constitue même parfois le sujet de moqueries auprès de certains théoriciens prétendument éclairés ${ }^{5}$. L'opprobre pesant sur l'hypothèse de Whorf déborda rapidement le cercle des initiés et se propagea telle une traînée de poudre à la population en général6, revêtant ainsi parfois l'aspect peu enviable d'une «légende urbaine» : de nos jours les sites Internet ayant pour raison d'être de ridiculiser la soi-disant hypothèse de Whorf foisonnent?.

\section{Comment cette hypothèse (parfois même subtilement hissée au rang}

ibid., "How relativistic are Humboldt's "Weltansichten»?" dans Pütz et Verspoor, Explorations..., op. cit., p. 25-44. Les divers travaux explicatifs visant à cerner et préciser le contenu de l'hypothèse de Whorf ne sont pas demeurés des caput-mortuum. Conformément à cela je limite, pour les fins poursuivies dans ce texte, la portée de l'expression "relativité linguistique» à l'utilisation qu'en a faite Whorf.

3. «Indeed, for some 25 years (at least from the late 1950 s to the late 1970 s) it was exceedingly hard to find a good word on behalf of Whorf in hardnosed, quantitative, experimental social science circles per se, or in the philosophical-theoretical circles derived from and influenced most directly by the social scientists." Joshua A. Fishman, "Whorfianism of the Third Kind : Ethnolinguistic Diversity as a Worldwide Societal Asset (The Whorfian Hypothesis : Varieties of validation, confirmation, and disconfirmation II)". Language Soc. 11, (1982) p. 1-14, p. 1.

4. Pour un examen en bonne règle du terme "pseudo science" (fausse science) consulter Karl R. Popper, Conjectures and Refutations : The Growth of Scientific Knowledge. Londres, Routledge \& Kegan Paul, 1962. Compulser également du même auteur The Logic of Scientific Discovery, New York, Basic Books, 1959, chapitre I section 4.

5. Je pense en particulier au célèbre livre de Geoffrey K. Pullum intitulé The great Eskimo vocabulary hoax and other irreverent essays on the study of language. Chicago, University of Chicago Press, 1991. II semble bien que Pullum, suivant les brisées de L. Martin, combatte davantage une figure mythique, ou plutôt caricaturale, de l'hypothèse de Whorf. Voir L. Martin, "Eskimo words for snow: a case study in the genesis and decay of an anthropological example", American Anthropologist 88, № 2, (1986), p. 418-23. De là la nécessité de bien cerner la teneur de ladite hypothèse afin de ne pas partir en guerre contre des moulins à vent. À ce propos voir Penny Lee, The Whorf Theory Complex : A Critical Reconstruction. Amsterdam et Philadelphie, John Benjamins, 1996; ibid. "When is "linguistic relativity" Whor's linguistic relativity?", dans Pütz et Verspoor, Explorations..., op. cit., p. 45-68.

6. Voir par exemple le jugement dévastateur porté par le réputé chercheur d'origine québécoise Steven Pinker contre l'hypothèse de Whorf dans son best-seller intitulé The Language Instinct. New York, William Morrow, 1994, p. 59-64.

7. Parmi les sites Internet voués à railler l'hypothèse de Whorf, l'exemple des termes inuits (eskimos) désignant la neige (exemple introduit à l'origine par Franz Boas) remportent la palme. Voir particulièrement le site hilarant de Phil James au site : http://www.word.com/gigo/eskimo/ 
L'hypothèse de Whorf s'applique-t-elle à la philosophie?

Brève réflexion sur les heurs et malheurs du rapport de la langue à la culture avec la philosophie comme toile de fond

gratifiant de "théorie» par quelques disciples irréductibles ${ }^{8}$ ) en est-elle venue si rapidement - soit en l'espace d'une décennie - à subir un tel discrédit? Moult éléments expliquent cette rapide «descente aux enfers» de l'hypothèse whorfienne. D'une part les efforts conjugués (mais pas nécessairement concertés) de philosophes ${ }^{9}$ tels que Max Black ${ }^{10}$, Lewis S. Feuer ${ }^{11}$, Charles Landesman ${ }^{12}$, Rulon Wells ${ }^{13}$ ou, à un tout autre niveau, Adam Schaff'14 -, et d'autre part l'émergence d'un nouveau courant linguistique - la grammaire générative de Noam Chomsky (courant qui comme on le sait allait tout balayer sur son passage en révolutionnant la discipline et en accordant une place de choix aux universaux de langage ${ }^{15}$

8. Il faut inévitablement compter parmi ceux-ci l'intransigeant anthropo-linguiste américain Dell Hymes qui, contre vents et marées, persista à propager une version forte de l'hypothèse de Whorf. Voir par exemple, Dell Hymes "On typology of cognitive styles in language (with examples from Chinookan)". Anthropological Linguistics, 3, (1961), p. 2-54; Ibid., "Two types of linguistic relativity (with examples from Amerindian ethnography)", dans W. Bright (ed.), Sociolinguistics, Proceedings of the UCLA sociolinguistics conference, 1964, La Haye, Mouton, 1966, p. 114-67; Ibid., Foundations in sociolinguistics : an ethnographic approach, Philadelphie, University of Pennsylvania Press, 1974.

Tout récemment Ocke-Schwen Bohn a affirmé sa foi indéfectible envers le statut épistémologique de la relativité linguistique. "For a speech scientist, linguistic relativity is not a hypothesis, "it's a fact"» (Je souligne). Ocke-Schwen Bohn, "Linguistic Relativity in Speech Perception. An overview of the Influence of Language Experience on the Perception of Speech Sounds from Infancy to Adulthood", dans Susanne Niemeier et René Dirven, Evidence..., op. cit., p. 1-28.

9. I.M Schlesinger soutient que ce sont davantage des conceptions philosophiques que de simples résultats empiriques qui ont précipité la chute de l'hypothèse de Whorf. Schlesinger, “The Wax..." op. cit, p. 7.

10. Max Black, "Linguistic Relativity: The Views of Benjamin Lee Whorf", The Philosophical Review, 68, (1959), p. 228-238; Ibid. "Some troubles with Whorfianism", dans S. Hook (ed.), Language and Philosophy : A Symposium. New York, New York University Press, 1969 , p. 30-35.

11. Lewis S. Feuer, "Sociological Aspects of the Relation between Language and Philosophy", Philosophy of Science, 20, (1953), p. 86.

12. Charles Landesman, "Does Language embody a Philosophical Point of View?», Review of Metaphysics, 14, (1961), p. 617-636.

13. Rulon Wells, "What has linguistics done for Philosophy?", The Journal of Philosophy, 59, (1962), p. 697-708.

14. Adam Schaff, Langage et connaissance, Éd. Anthropos, 1967. Voir également de l'Espagnol Lorenzo Peña, "La critica de Stalin al relativismo lingüistico". (La critique stalinienne du relativisme linguistique) au site :

http://er.users.netlink.co.uk/biblio/stalin/linguist/interling.htm.

15. Notons qu'à la même époque, mais en restant à l'écart des théories révolutionnaires proposées par Chomsky, J. H. Greenberg s'est mis en quête de pourchasser les universaux de langage. Voir J.H. Greenberg. Universals of Language, Cambridge, Mass. : MIT Press, 1963. Les efforts entrepris par Greenberg débouchèrent, au cours des années 70, sur un ouvrage monumental. Voir J.H. Greenberg, C.A. Ferguson et E.A. Moravcsik (ed.) Universals of Human Language (4 tomes). Standford, Standford University Press, 1978. 
puis, en privilégiant la syntaxe au détriment de la sémantique) - semblent avoir porté un coup de grâce à l'hypothèse de la relativité linguistique. Les assauts successifs portés contre certains châteaux forts de la relativité linguistique ${ }^{16}$ ont créé une brèche par laquelle se sont prestement engouffrées des théories étayées par des investigations empiriques ${ }^{17}$ discrédi-

16. Je pense en particulier aux termes désignant les couleurs. Perçu dès le début comme un allié "naturel" de la relativité linguistique, le discours sur les couleurs devint après la célèbre étude menée par Berlin et Kay (1969) le parangon de l'existence d'universaux dans le langage. Notons qu'ultérieurement Kay se ravisa et on peut même interpréter son article de 1984 comme exprimant de la sympathie envers l'hypothèse de Whorf. Voir B. Berlin et P. Kay, Basic color terms : their universality and evolution, Berkeley, University of California Press, 1969;

Paul Kay et Willett Kempton, "What is the Sapir-Whorf hypothesis?", American Anthropologist, 86, (1984), p. 65-79. Pour un traitement récent de la problématique particulière associée au langage des couleurs voir de Gordon Lyon, "Language and Perceptual Experience", Philosophy, 74, (1999), p. 515-34. Le phénomène de l'émergence de nouvelles théories pour expliquer la diversité linguistique relativement à la catégorisation (lexicalisation) des termes se rapportant aux couleurs (par exemple la "Vantage theory" de Robert E. MacLaury) doit être perçu comme un appui potentiel à l'endroit de l'hypothèse de Whorf. Voir Robert MacLaury, "Vantage theory", dans John R. Taylor et Robert E. MacLaury (ed.), Language and the Cognitive Construal of the World, Trends in Linguistics 82, Berlin/New York, Mouton de Gruyter, 1995, p. 231-76; Keith Allan, "Colors and Vantage Theory", Language-Sciences 21; No 4, (Oct. 1999), 4 , p. 449-460; J. van Brakel "The Ignis Fatuus of semantic universalia : the case of colour", British Journal for the Philosophy of Science, 45, (1994), p. 770-83; John A. Lucy, "The linguistics of "color"”, dans C. Hardin et L. Maffi (ed.), Color Categories in Thought and Language. Cambridge, Cambridge University Press, 1997, p. 320-346.

Dan Slobin, pour sa part, prend ses distances par rapport à l'étude comparative des termes désignant des couleurs en soutenant que ceux-ci sont trop déterminés par la biologie. Le domaine d'investigation privilégié par Slobin (les verbes de mouvements) avalise la position de Whorf en misant sur le slogan "Thinking for speaking". Dan I. Slobin, "From "thought and language" to "thinking for speaking"», dans John J. Gumperz et Stephen C. Levinson (ed.), Rethinking Linguistic Relativity, Studies in the Social and Cultural Foundations of Language 17, Cambridge, Cambridge University Press, 1996, p. 70-96; Ibid., "Verbalized events : A dynamic approach to linguistic relativity and determinism" dans Niemeier et Dirven, Evidence..., op. cit. p. 107-38.

Dans un tout autre registre, E. Malotki, un élève de Helmut Gipper, s'ingénia à démontrer l'inexactitude des analyses whorfiennes portant sur les notions temporelles des indiens Hopi. Voir E. Malotki, Hopi Time : a linguistic analysis of the temporal categories in the Hopi language, Berlin, Mouton, 1983. Les résultats de Malotki ne sont toutefois pas acceptés par une majorité de linguistes.

Notons que ces domaines respectifs recoupent ce que Lucy désigne comme des Domain-Centered et Structure-Centered Approaches. John A. Lucy, "Linguistic Relativity». Annual Review of Anthropology, 26, (1997), p. 291-312.

17. Voir le stimulant essai de Csaba Pléh, «Hozzájárulhatnak-e az empirikus pszichológiai kutatások a nyelv-gondolkodás viszony filozófiai problémájának megoldásához?» (Les investigations empiriques en psychologie peuvent-elles contribuer à résoudre les problèmes philosophiques de la relation langue-pensée?) dans Katalin Neumer (ed.), Nyelv, gondolkodás, relativizmus (Langue, pensée, relativisme), Budapest, Osiris, 1999, p. 35165. 
tant pour un moment le bien-fondé de la relativité linguistique ${ }^{18}$. On pourrait également alléguer que l'érosion progressive de l'intérêt porté par la communauté philosophique envers le langage ordinaire (et par ricochet à la philosophie du langage en général) et le subit engouement que cette même communauté démontra envers la philosophie de l'esprit a exercé un rôle non négligeable dans l'effondrement de l'hypothèse de Whorf ${ }^{19}$. II serait évidemment présomptueux d'isoler un de ces éléments et de lui attribuer un rôle prépondérant dans la déchéance de l'hypothèse concernée. C'est plutôt l'ensemble de ces causes - ce que nous pourrions nommer une conjoncture favorable - qui a contribué à vouer l'hypothèse de Whorf aux gémonies 20 .

Quoi qu'il en soit, approximativement depuis le début des années 90 , un phénomène intéressant se dessine : l'hypothèse de la relativité linguistique de Whorf (expurgée cependant de son vocabulaire (ésotérique?!) peu conforme aux canons de la science linguistique contemporaine) $)^{21}$ semble vouloir renaître de ses cendres et partant son blason resplendit d'un éclat rappelant la magnificence d'antan. Contre toute attente, la revivification de l'hypothèse si longtemps proscrite fait d'un programme de recherche que l'on jugeait moribond (ou dégénératif pour utiliser un vocable cher à Imre Lakatos) un programme de recherche productif (ou prospectif) sur un plan heuristique. Hormis les efforts louables d'individus tels John A. Lucy22 et Penny Lee ${ }^{23}$, il faut également mentionner la paru-

18. Voir John A. Lucy, "Linguistic relativity", op. cit.

19. Voir de Sandra Laugier, Du réel à l'ordinaire. Quelle philosophie du langage aujourd'hui?, Paris, Librairie Philosophique J. Vrin, 1999. Notons au passage qu'un influent texte de référence en philosophie analytique du milieu des années 80 persiste à mettre l'hypothèse de Whorf (ou plutôt un travestissement de celle-ci) au ban. Voir M. Devitt et K. Sterelny, Language and Reality : an Introduction to the Philosophy of Language, Oxford, Basil Blackwell, 1987.

20. Pour une étude critique des idées fausses ayant contribué à précipiter la chute de l'hypothèse de Whorf voir Danny Keith Hawkmoon Alford, "The demise of the Whorf hypothesis : A major revision in the history of linguistics". Proceedings of the 4th Annual Meeting of the Berkeley Linguistics Society, Berkeley, p. 485-99.

21. La laxité du vocabulaire et des formulations de Whorf ajoutent à la difficulté de comprendre la teneur de sa théorie et en fait une sorte de sabir. Son attachement aux théories de Carl Jung, sa dévotion à la pratique du zen, sa sympathie envers le mouvement théosophique, ne sont sûrement pas étrangers au caractère sibyllin de ses écrits théoriques. Un commentateur ne se gêne guère pour affirmer que "Whorf schreibt unklar und verworren". (Whorf écrivait d'une façon confuse et embrouillée). Voir Kay González Vilbazo, "Sapir-Whorf-Hypothese», Linguistik-Forum WS 1994/5, Köln, 06.12.94. Voir également Max Black, "Linguistic Relativity...", op. cit., p. 228.

22. John A. Lucy, Language diversity..., op. cit.; Ibid. Grammatical categories and cognition : a case study of the linguistic relativity hypothesis, Cambridge, Cambridge University Press, 1992.

23. Penny Lee, The Whorf Theory..., op. cit. 
tion d'un influent ouvrage collectif dirigé par John J. Gumperz et Stephen C. Levinson (1996) ${ }^{24}$, ainsi que la tenue d'un congrès en avril 1998, à Duisburg, destiné à donner un nouvel éclairage aux théories de Humboldt et Whorf25. Les textes précités jouent un rôle à la fois séminal et instrumental dans la renaissance des études visant à infirmer ou, selon le cas, confirmer l'hypothèse de Whorf (ou certains aspects de celle-ci) et sont en passe de devenir des écrits phares guidant une nouvelle génération de chercheurs désireux d'évaluer (à son juste mérite et sans idée préconçue) l'hypothèse de Whorf. Le mot d'ordre toutefois ne semble plus être "d'exhumer", au nom d'une certaine orthodoxie, le "cadavre" de Whorf mais bien de réévaluer les thèses de celui-ci afin de les accommoder aux nouvelles réalités caractérisant la linguistique contemporaine (par exemple la grammaire cognitive de Ronald Langacker ${ }^{26}$, la grammaire culturelle de Gary Palmer27, le "Natural Semantic Metalanguage" d'Anna Wierzbicka28, les nouvelles théories sémantiques ${ }^{29}$, l'écolinguistique ${ }^{30}$, etc.). Le nombre croissant d'expériences empiriques visant à confirmer certains aspects de la relativité linguistique (ou prosaïquement de l'influence du langage sur la pensée) attestent la vitalité de ce domaine d'investigation et constitue, rétrospectivement, un événement majeur des années 90 .

Qu'en est-il maintenant du statut de l'hypothèse de Whorf en philosophie? En dépit de son air éminemment suspect, exacerbé par la mise à nu de la «linguistic fallacy» de Max Black, l'hypothèse de Whorf (ou une ver-

\section{John J. Gumperz et Stephen C. Levinson, Rethinking..., op. cit.}

25. Les allocutions prononcées lors du $26^{\mathrm{e}}$ Symposium international de la LAUD (Linguistic Agency University Duisburg), à l'université Gerhard Mercator, sont colligées dans les volumes dirigés par Susanne Niemeier et René Dirven, Evidence..., op. cit. et Martin Pütz et Marjolijn Verspoor, Exploration..., op. cit.

26. Ronald W. Langacker, Foundations of cognitive grammar Vol. 1, Standford, Standford University Press, 1987; Ibid. Vol. 2, 1991.

27. Gary B. Palmer, Toward a Theory of Cultural Linguistics, Austin, University of Texas Press, 1996.

28. Anna Wierzbicka, Semantics, Culture, and Cognition. New York, Oxford University Press, 1992; Understanding Cultures Through their Keywords : English, Russian, Polish, German, and Japanese. New York/Oxford, Oxford University Press, 1997. Nick J. Enfield déplore, à juste titre, la méconnaissance des théories de Wierzbicka dont font preuve les auteurs traitant du problème de la relativité linguistique. Voir Nick J. Enfield, "On Linguocentrism", dans Pütz et Marjolijn, Explorations..., op. cit., p. 125-147.

29. Les nouvelles théories sémantiques sont passées en revue par Michael A. K. Halliday dans "New ways of analyzing meaning" dans M. Pütz (ed.), Thirty Years of Linguistic Evolution, Amsterdam et Philadelphie, John Benjamins, p. 59-96.

30. Peter Mühlhäusler, "Humboldt, Whorf and the Roots of Ecolinguistics», dans Pütz et Verspoor, Exploration..., op. cit., p. 89-99. 
sion plus ou moins orthodoxe de celle-ci) a été manifestement à l'œuvre chez Paul K. Feyerabend ${ }^{31}$ (mais latente chez Thomas Kuhn) ${ }^{32}$ et elle n'est visible qu'en filigrane chez Quine ${ }^{33}$. Notons à la volée que ce trio d'épistémologues (qu'une certaine intention commune rapproche) faisait de la notion de relativité la pierre angulaire de leur programme philosophique respectif, voilà ce qui expliquerait, en partie, la rémanence de certaines thèses d'inspiration whorfienne dans leurs écrits. Nous pouvons toutefois compter sur le bout des doigts les études philosophiques récentes portant de près ou de loin sur ladite hypothèse. Les rares études qui osent le faire mentionnent ou bien "accessoirement» l'hypothèse de Whorf afin de mettre celle-ci soit en opposition, soit en relation avec une autre position (principalement celle de Wittgenstein) jugée prédominante dans la comparaison ${ }^{34}$ ou tentent d'évacuer carrément la pertinence d'une interrogation de ladite hypothèse en critiquant d'emblée le présupposé épistémologique sur lequel s'érige celle-ci. Ainsi la stimulante querelle entre Quine et Davidson ${ }^{35}$ sur le dualisme schème/contenu a eu pour effet d'éloigner davantage la communauté philosophique de l'urgence de

31. Voir en particulier Against Method. Outline of an Anarchistic Theory of Knowledge. Londres, Verso, 1975. p. 223-24 et l'appendice 5. L'empressement partisan de Feyerabend à utiliser une "version trop forte" de la version de l'hypothèse de Whorf explique peut-être le lapsus calami qu'il commet dans son magnum opus en persistant à écrire Whorff au lieu de Whorf. La mauvaise graphie apparaît dès 1962 dans "Explanation, Reduction and Empiricism" et se perpétue jusqu'à l'édition de 1988 de Against Method. L'erreur est heureusement corrigée dans Farewell to Reason, Londres, Verso, 1987.

32. Kuhn fait une allusion timide aux théories de Whorf dans la préface de The Structure of Scientific Revolutions, $2^{e}$ éd., Chicago, University of Chicago Press, 1970, p. vi.

33. Quine mentionne, en passant, l'hypothèse de Whorf dans quelques uns de ses textes primitifs. Ceux-ci sont: "The Problem of Meaning in Linguistics" (1951), dans From a Logical Point of View, Harvard University Press, 1953, $2^{\mathrm{e}}$ (1961), p. 47-64, p. 61; Ibid. Word and Object, Cambridge, MIT Press, 1960, $g^{e}$ 1975, p. 71. Étonnamment bien que la dette de Quine envers Whorf semble aller de soi (c'est-à-dire "usage» de la notion de holisme, de structuralisme, de relativité (linguistique pour l'un, ontologique pour l'autre), d'incommensurabilité, etc.), la "mention" du nom de Whorf disparaît des textes de Quine à l'aube des années 60 , c'est-à-dire au moment même où Whorf voit son étoile pâlir dans le firmament philosophique. Voir également Sandra Laugier, L'anthropologie logique de Quine, Paris, Vrin, 1992; Ibid. "Relativité linguistique, relativité anthropologique", Histoire, épistémologie, langage, 18, № 2, (novembre 1996), p. 45-73.

34. M. Kienpointner, "Whorf and Wittgenstein. Language, World View and Argumentation", Argumentation, 10, № 4, (novembre 1996), p. 475-94; Ranjit Chatterjee, "Reading Whorf through Wittgenstein : A Solution to the Linguistic Relativity Problem". Lingua, 6, 7, (Sept. 1985), p. 37-63. Voir également Katalin Neumer, «Nyelv, Gondolkodás, relativizmus. Filozófiai vizsgálódás" (Langue, pensée, relativisme. Une enquête philosophique), dans ibid., Nyelv, Goldolkodás,... op. cit., p. 13-34.

35. Querelle inaugurée par l'article séminal de Donald Davidson, "On the Very Idea of a Conceptual Scheme», Proceedings and Addresses of the American Philosophical 
reconsidérer à sa valeur propre la pertinence de l'hypothèse de Whorf en refocalisant l'attention sur un problème où celui-ci était exclu comme joueur. II en résulte une situation où l'hypothèse de Whorf est jugée perdante avant même qu'elle soit de nouveau examinée. Le plus déplorable dans tout cela c'est que la version de l'hypothèse de Whorf critiquée jusqu'à présent n'est qu'une vulgaire simplification imitant d'une façon grotesque la véritable version proposée à l'origine par le linguiste amateur américain. Je crois que le moment est venu d'inscrire un da capo au dessus de l'état actuel de la problématique de la relativité linguistique afin de donner une nouvelle chance à Whorf. On peut espérer que le corollaire suivant s'avère vrai : le fait de reconsidérer $a b$ ovo l'hypothèse de Whorf aura pour conséquence de relancer d'une façon inattendue les recherches philosophiques sur la relativité linguistique et permettra de préciser davantage les liens problématiques reliant la langue à la culture et à la pensée.

L'objectif poursuivi dans ce texte est le suivant : Il s'agit dans un premier temps d'introduire des éléments visant à démontrer la pertinence de l'hypothèse de la relativité linguistique de Whorf à l'aide d'exemples nous permettant de croire à l'existence d'une influence du langage sur la pensée (ou la culture). On tentera en second lieu d'analyser d'une façon critique la possibilité d'une extension - ou d'une généralisation - de l'hypothèse de Whorf à la philosophie. En d'autres termes le fait de parler (ou d'écrire) une certaine langue nous permet-il de croire que cela influence (ou détermine) la formulation d'une philosophie donnée? Afin d'évaluer la portée de cette hypothèse de départ nous prenons comme point d'appui un texte engagé à défendre l'hypothèse à évaluer. L'intitulé du texte en question est «Linguistic Relativity in French, English, and German Philosophy36». Après avoir critiqué la possibilité d'une généralisation de la thèse whorfienne à la philosophie nous proposons en contrepartie une variante de la théorie whorfienne (une hypothèse de Whorf «réaménagée» oserions-nous dire) afin d'illustrer une influence possible du langage sur la philosophie.

Association, 47, (1974), reproduit dans ibid., Inquiries into Truth and Interpretation, Oxford, Clarendon Press, 1984, p. 183-98. Pour une lecture de l'approche davidsonnienne en regard de l'hypothèse de Whorf voir W.A. Berriman, "Alternative Conceptual Schemes", Metaphilosophy, Vol. 9, № 3 et 4, (juillet/octobre 1978), p. 226-232.

36. William Harvey, "Linguistic Relativity in French, English, and German Philosophy", Philosophy Today (Été 1996) p. 273-288. 


\section{La bougainvillée et une «talle» ${ }^{37}$ de fraises des bois.}

Le regretté Octavio Paz (1914-1998), un écrivain mexicain de glorieuse mémoire, rapporta dans un de ses textes les plus notoires les paroles d'une de ses amies en proie au désarroi résultant de la subite prise de conscience de l'étrangeté de sa langue maternelle ${ }^{38}$ transplantée dans un lieu, un environnement, un Lebenswelt - ou pour utiliser un vocable cher à Jacob von Uexkhüll un "milieu» (Umwelt) -, fondamentalement différent (tant sur l'aspect géographique que culturel) de celui auquel elle était habituée depuis sa naissance. Tenant compte de l'importance dudit passage pour notre propos à venir, je prends la liberté de le citer en entier :

Je me souviens, par exemple, de ce que me répondit une amie à qui je faisais remarquer la beauté de Berkeley : «Mais oui, c'est très beau; pourtant je n'arrive pas à comprendre... Ici, même les oiseaux parlent anglais. Et comment pourraient-elles me plaire ces fleurs dont je ne connais pas le véritable nom, le nom anglais, un nom qui ne fait plus qu'un avec les couleurs et les pétales, un nom qui est la chose même? Quand tu dis bougainvillées, tu penses à celles que tu as vues dans ton village, grimpant sur un frêne, humides et liturgiques, ou sur un mur, certain soir, dans une lumière argentée. La bougainvillée forme une partie de ton être, de ta culture, c'est ce que tu te rappelles après l'avoir oublié. Ici, tout est très beau, mais ce n'est pas à moi : ce que disent le prunier et les eucalyptus ce n'est pas à moi qu'ils le disent, ce n'est pas pour moi39.

Comme le laisse entrevoir la citation de Paz, la croyance populaire récupérée et alimentée par une certaine tradition philosophique fusant de l'étrange doctrine cratyliste du Socrate de Platon 40 - tend à associer depuis des temps immémoriaux les mots et les choses comme si un mystérieux lien mimétique, une copula, unissait les mots aux objets

37. "Talle», canadianisme : $n$. f. touffe de plantes d'une même espèce. Dictionnaire Bélisle de la langue française au Canada. Montréal, Société des Éditions Leland, 1958.

38. Dans une chanson intitulée Língua (Langue), le chanteur brésilien Caetano Veloso, coryphée du mouvement "tropicaliste", joue habilement (avec la licence que seuls les poètes peuvent se permettre) sur les notions de patrie, de langue maternelle et de fraternité : “A língua é minha pátria/ $E$ eu não tenho pátria : tenho mátria/ $E$ quero frátria». (La langue est ma patrie/ Je n'ai pas de patrie : j'ai une "matrie"/et je désire une «fraterie"). Caetano Veloso, Velô, Polygram Brasil, 1984. Sur l'aspect synchronique du concept de "langue maternelle» voir Einar Haugen, "The «mother tongue»", dans Robert L. Cooper et Bernard Spolsky, The Influence..., op. cit., p. 75-84.

39. Octavio Paz, Le labyrinthe de la solitude, trad. française de Jean-Clarence Lambert, Paris, Gallimard, 1976, p. 19-20.

40. Voir Gérard Genette, Mimologiques. Voyage en Cratylie. Paris, Éditions Du Seuil, 1976. 
nommés ${ }^{41}$. Le malaise de la locutrice de Paz, manifestement mal acclimatée à son nouvel environnement californien, évoque cette perte du sentiment "d'enracinement» liant les choses à leur lieu d'origine ou, en d'autres termes, la perception aiguë que les mots n'assureraient plus une "dénomination juste» des objets du monde mais qu'ils seraient le résultat d'un "choix arbitraire" variant synchroniquement avec le choix d'une langue (système symbolique) d'énonciation ${ }^{42}$. Le caractère éminemment arbitraire du signe linguistique - un lieu commun depuis Saussure ${ }^{43}$ - signale avant toute chose la plasticité des lexèmes qui, outre la multiplicité des phonèmes utilisés dans les différentes langues pour les exprimer verbalement, servent également à catégoriser, selon des arrangements dissemblables et en vertu des intérêts et des points de vue privilégiés par les locuteurs, différents référents. En quoi peut-on être certain que le symbolisme associé aux bractées de la buganvilla, se dodelinant langoureusement sous le doux alizé d'une quelconque llano sud-américaine et formant "une partie de ton être, de ta culture", désigne la même chose (intentionnellement) que la bougainvillée exposée au Jardin botanique à Montréal? L'exemple de Paz me semble excellent pour introduire la notion de relativité linguistique car sous le couvert d'un terme anodin - tirant, de surcroît, sa dénomination d'un nom propre (présumant faussement la justesse d'une dénomination juste) - une myriade de connotations culturelles réussit tout de même à émerger. En effet l'excellence de l'exemple de Paz est rehaussée par le fait que le terme "buganvilla" est facilement "repérable», «lisible», "déductible», dans la majorité des autres langues. Comme on le sait la bougainvillée tire son appellation d'origine du nom patronymique du navigateur français Louis Antoine de Bougainville. Ceci étant dit on comprend aisément dès lors que les termes bougainvillea ${ }^{44}$, buganvillea ${ }^{45}$, buganvilia ${ }^{46}$ désignent (en principe) la même chose. Or la question qu'il faut évidemment se poser c'est de savoir si ces termes,

41. Voir Katalin Neumer, "Les mots et les choses. Denken, Sprechen, Schreiben in Herders Philosophie», (Les mots et les choses. Penser, parler et écrire dans la philosophie de Herder) dans : Wolfram Hogrebe (ed.), Filosofia Hungarica. Profile zeitgenossischer philosophischer Forschung in Ungarn, (Philosophie hongroise. Profils de la recherche philosophique contemporaine en Hongrie) Verlag Konigshausen \& Neumann 2001, p. 155-166. (sous presse)

42. Voir Edmond L. Wright, "Arbitrariness and Motivation : A New Theory", Foundations of Langage, 14, (1979), p. 505-523.

43. Anticipé toutefois par Gerauld de Cordemoy, Discours physique de la parole (1677). Édition en fac-similé, Herbert Brekle (ed.), Stuttgart, Friedrich Frommann Verlag, 1970.

44. En allemand, anglais, polonais, suédois, norvégien, danois, etc.

45. Italien.

46. Portugais. 
semblables en apparence, connotent effectivement la même chose? Un élément de réponse se pointe aussitôt que l'on mentionne l'équivalent malais ${ }^{47}$ du terme bougainvillée : pohon bugenvil. Ainsi lorsqu'un Malais (ou un Indonésien) parle d'une bougainvillée, il doit nécessairement faire précéder le mot bugenvil (qui comme on l'aura deviné est une approximation phonétique du mot bougainvillée) d'un "déterminatif de classe»: en l'occurrence pohon. Or pohon désigne en malais la classe des arbres; ce qui nous laisse croire que les Malais catégorisent la bougainvillée $e^{48}$ davantage comme un arbre (arbuste) que comme une plante ornementale (comme c'est le cas par exemple pour les hispanophones tel que le démontre clairement l'exemple de Paz). En comparaison, le mot orchidée, qui à l'instar de la bougainvillée désigne également une plante ornementale prisée pour ses fleurs, se traduit en malais par bunga anggerik. Comme pour le terme pohon bugenvil, le terme bunga désigne ici un déterminatif de classe utilisé pour nommer, cette fois-ci, la classe des fleurs (bunga); fleur qui sera plus spécifiquement désignée par l'adjonction d'une espèce précise : anggerik (orchidée). Conformément à cet exemple impliquant d'une part la bougainvillée (différant de la catégorisation usuelle des Européens) et, d'autre part, l'orchidée (identique à la catégorisation qu'en donnent les Européens) nous sommes en mesure de constater que les Malais et les Européens (ou soit les langues regroupées par Whorf sous le vocable de SAE "Standard Average European) 49 catégorisent différemment les végétaux. Ainsi, sans le savoir, Paz nous introduit d'une façon magistrale au problème de la relativité linguistique ${ }^{50}$. La

47. Le Bahasa Malaysia est la langue nationale (bahasa kebangsaan) de la Malaisie en plus du chinois. Le Malais s'apparente à la langue indonésienne (Bahasa Indonesia).

48. Je crois que les Malais désignent la bougainvillée par un terme correspondant à la classe des arbres en raison du fait que la bougainvillée est une plante grimpante, c'est-à-dire quelque chose qui se dresse verticalement sur une certaine hauteur (à l'instar d'un arbre) par rapport au locuteur. Le système de référence serait donc conçu en regard de la disposition des objets nommés vis-à-vis de la position du locuteur.

49. Whorf, "The Relation of Habitual Thought and Behavior to Language", Ibid., Language..., op. cit., p. 138.

50. L'exemple introduit par Paz nous fait immédiatement penser à la comparaison établie par Herder entre la langue et un jardin. "Jedes Buch ist ein Beet von Blumen und Gewächsen; jede Sprache ein unermäßlicher Garten voll Pflanzen und Bäume...” (Chaque livre est un parterre de fleurs et de végétaux; chaque langue un immense jardin bondé de plantes et d'arbres...), Johann Gottried Herder, Über die neuere deutsche Literatur, dans Wolfgang Pross (ed.), Johann Gottfried Herder Werke Band I, Munich, Carl Hanser Verlag, 1984, p. 66-354, p. 75.

L'idée d'associer le caractère arbitraire du nom d'une fleur à l'acte de dénomination semble être un thème récurrent de la littérature occidentale. Ainsi, par exemple, la jeune poétesse suédoise Pia Paulina Nykänen (née en 1970) exhale un soupir de découragement et murmure "Bleka blomma/ var är ditt namn"? (Pâle fleur/ où est ton nom). Strophe tirée du poème "Blomning, juni» (Floraison, juin) de son premier recueil intitulé 
perception fondamentale du phénomène de la relativité linguistique passe «initialement" par la perception d'une différence au niveau de la manière dont les lexèmes des langues respectives catégorisent différemment le contenu de l'expérience sensible. Benjamin Lee Whorf s'empresse d'ailleurs de mettre en relief cet aspect foncier de l'hypothèse de la relativité linguistique : "Languages differ not only on how they build their sentences but also in how they break down nature to secure the elements to put in those sentences. This breakdown gives units of the lexicon ${ }^{51}$ ".

Comme l'indique précisément la strophe du poème de Tomas Thøfner citée in limine, les substantifs (morphèmes lexicaux) ${ }^{52}$ désignant des objets physiques de grandeur moyenne (middle size dry goods) constituent le socle à partir duquel s'érige le langage ${ }^{53}$. L'hypothèse de la relativité linguistique émane du principe suivant lequel des cultures hétérogènes catégorisent différemment le contenu de l'expérience car ce contenu varie en fonction même de leur environnement respectif. Le manque de correspondance (directe et univoque) entre les langues provient donc de cette différence environnementale basale. La neige constitue un élément essentiel de l'environnement des Inuits (dixit Boas), partant ceux-ci maîtrisent un vocabulaire apte à exprimer les nuances qu'ils jugent nécessaires et qui, en retour, a une incidence sur leur vie quotidienne. De la même manière un fermier danois possédera un vocabulaire adapté à son environnement; il ne faut guère se surprendre si un citadin, parvenant tout juste à distinguer un cheval d'un mulet, désigne un cheval par le simple terme générique "hest» (cheval), alors qu'un fermier du Jutland oriental utilisera une panoplie de termes pour exprimer des

Herbarium, Stockholm, Bonnier, 2000. Nous pouvons faire remonter cette image à cette strophe de William Shakespeare : "What's in a name? that which we call a rose/By any other name would smell as sweet». Voir Roméo et Juliette 2.2.43.

51. Whorf, "Languages and Logics" dans Ibid., "Language...", op. cit., p. 233-45, p. 240.

52. A. Deigman, M. Knowles, J. Sinclair et D. Willis (ed.), Lexis, Birmigham, Birmigham University Press, 1995.

53. Cette conception concorde avec la doctrine quinienne de l'apprentissage des langues naturelles (qu'elles soient maternelles ou secondes). Voir W. V. Quine, "Speaking of Objects", dans Ibid., Ontological Relativity and Other Essays, New York/Londres, Columbia University Press, 1969, p. 1-25; Voir également "Things and Their Place in Theories", dans Ibid., Theories and Things, Cambridge/Londres, Harvard University Press, 1981, p. 1-24. L'état actuel de la recherche sur l'acquisition d'une langue seconde (SLA : Second Language Acquisition) tend cependant à distinguer ce type d'apprentissage de l'apprentissage du langage maternel. Voir R. Ellis, The Study of Second Language Acquisition, Oxford, Oxford University Press, 1994. Voir également le concept du «The Original Word Game" de Roger Brown, dans Words and Things, New York, The Free Press, 1958. 
nuances qui échappent au citadin 54 : le paysan ne dira jamais "hest" mais plutôt, selon le cas, hingst (étalon), hoppe (jument), vallak (hongre), føl (poulain), plag (jeune cheval), skimmel (cheval pommelé), pasgænger (cheval ambleur), remonte (cheval de remonte), stikkelhåret (cheval baie), krikke (rosse), en plus d'identifier la race d'origine des chevaux : belge, jutlandais, arabe, etc. $55 \mathrm{Ou}$ bien encore, en parcourant à pied la verdoyante Scanie (Suède), on apprendra avec surprise que la fraise (jordgubbar) change de nom simplement en raison du fait qu'elle est cultivée ou sauvage; dans ce dernier cas on lui attribue en Suède le nom de smultron - une espèce vantée naguère par le grand Linné pour ses propriétés médicinales - tandis qu'en français et dans la majorité des autres langues européennes ${ }^{56}$ on se contente de nommer cette espèce fructifère en ajoutant soit le qualificatif "sauvage" : "fraise sauvage" (fran.), wild strawber$r y$ (angl.), wild Erdbeere (all.), vild jordbær (dan.), wild aardbei (néerl.), ou bien "des bois": fresas silvestres (esp.), fragole di bosco (ital.), umska jagoda (serbo-croate), lesní jahoda (tchèque), markjordbær (norvégien). ${ }^{57}$ Le finnois en revanche possède un terme unique pour désigner les fraises, qu'elles soient de culture ou des bois (sauvages) : mansikka. Cependant si on désire préciser que ce sont des fraises de culture il suffit de faire précéder le terme mansikka par le mot puutarha- (jardin) pour former par préfixation le terme : puutarhamansikka (fraise de culture). Le hongrois, également une langue de la famille finno-ougrienne, exige de préciser la nature de la provenance de la fraise: le terme "eper» (fraise) doit être précédé des mots földi ou kerti : földi eper pour les fraises sauvages et kerti eper pour les fraises de culture. Le portugais utilise également un seul terme pour désigner les fraises (de culture et des bois) : morango. Cet excursus en compagnie d'une espèce fructifère répandue nous permet d'illustrer la relation serrée qui existe entre les lexèmes d'une langue et la façon dont celle-ci subdivise le monde sensible en permettant

54. Ce type de relativité linguistique est désigné par Lucy comme de la relativité discursive (ou fonctionnelle). Voir également Dell Hymes, "Two Types...", op. cit.; L.S. Vygotsky (1930-34), Mind in Society. The Development of Higher Psychological Processes, M. Cole (ed et trad.), Cambridge, Harvard University Press, 1978 et ibid., Thought and Language (1934), Cambridge, MIT Press, 1987.

55. Exemple tiré d'un texte d'Ole Stig Andersen intitulé «Der er nok kun to rigtige ord for sne på grønlandsk». (II existe seulement deux mots justes pour désigner la neige au Groenland), au site http://www.olestig.dk/klummer/sne.html.

56. Le russe, à l'instar du suédois, possède deux mots pour désigner les fraises : klybnika pour les fraises sauvages et zemlyanika pour les fraises de culture. Le polonais fait de même : truskawka (fraise de culture) et poziomka (fraises des bois). Le lituanien permet deux constructions : soit_emuog_ (fraise, sans préciser si ce sont des fraises sauvages ou de culture) et mi_ko_emuog_ (afin de préciser qu'il s'agit de fraises des bois) ou bien bra_ké pour désigner uniquement les fraises de culture.

57. Mark = champ; fraises des champs. 
diverses constructions mentales mettant en relief différents aspects d'un objet jugé (perceptiblement) invariant58. Pour les uns (les Suédois) la "fraise" smultron est avant tout un cultivar portant déjà en elle le sceau de la culture (au double sens du mot) alors que pour d'autres - les Finnois par exemple - la fraise [mansikka] est avant tout un fruit sauvage; le poids de la culture se fait sentir dès que l'on précise qu'il s'agit d'une puutarhamaniskka. D'autres langues (par ex : le portugais) ne jugent pas nécessaire de distinguer entre l'espèce sauvage et celle cultivée. Ainsi le génie d'une langue réside principalement dans la manière dont elle s'y prend pour évoquer les objets se trouvant dans son environnement naturel59.

Cette constatation initiale constitue toutefois un élément trivial de l'hypothèse de Whorf60. Cette trivialité ne doit cependant pas nous faire perdre de vue que cela constitue un élément essentiel de l'apprentissage des langues étrangères (et par ricochet la découverte de la relativité linguistique). Quiconque se donne la peine d'apprendre une langue étrangère comprend immédiatement cela61. L'hypothèse de la relativité linguistique de Whorf va cependant au-delà de la simple constatation que les langues arrangent différemment leurs morphèmes lexicaux (rapport langue-langue)62. L'aspect novateur de l'hypothèse de Whorf réside davantage dans la tentative de faire correspondre des comportements non-linguistiques (culturels ou cognitifs) à des constructions langagières afin de démontrer une possible influence de ces dernières sur les premiers et par conséquent d'échapper au risque de circularité découlant du

58. Charles Hockett présente un exemple similaire en indiquant la différence entre la catégorisation des anglo-saxons des fraises et des fruits par rapport à la catégorisation des chinois (yángméi/tsauméi (fraise), et gwò. Voir Charles F. Hockett, "Chinese Versus English : An Exploration of the Whorfian Theses" dans Hoijer (ed.), Language in culture, op. cit., p. 106-123; p. 111-112.

59. Eugene H. Casad, "Seeing it in more than one way", dans John R. Taylor et Robert E. MacLaury (ed.), Language and the Cognitive Construal..., op. cit., p. 23-49.

60. Selon la typologie suggérée par Joshua Fishman cela constitue le premier niveau (le niveau langage-langage) de la division quadripartite qu'il introduit dans "Systematization...", op. cit., p. 326-329.

61. De fait Whorf soutient que la meilleure façon de prendre conscience de l'influence (larvée) qu'exerce notre propre langage sur notre manière de catégoriser le monde consiste à opposer notre façon de voir les choses à celle véhiculée par une autre langue. "...the best approach is through an exotic language, for in its study we are at long last pushed willy-nilly out of our ruts. Then we find that the exotic language is a mirror held up to our own." Whorf, "The Relation...", op. cit., p. 138.

62. Gregory Hadley, "Lexis and Cultures : Bound and determined?", Journal of Psycholinguistic Research, Vol. 26, № 4, (1997), p. 483-96. 
L'hypothèse de Whorf s'applique-t-elle à la philosophie?

Brève réflexion sur les heurs et malheurs du rapport de la langue à la culture avec la philosophie comme toile de fond

fait de comparer un langage avec un autre langage ${ }^{63}$. Afin d'introduire cette dimension 64 de l'hypothèse de la relativité linguistique de Whorf permettez-moi de citer de nouveau un exemple extrait de la littérature sudaméricaine.

Le romancier brésilien José Martiniano de Alencar (1829-1877) soucieux de valoriser la spécificité de la culture luso-brésilienne par rapport à celle des colonisateurs portugais - écrivit, avec une pointe d'humour certes, dans Sonhos d'Ouro (1872):

0 povo que chupa o caju, a manga, o cambuca e a jabuticaba, pode falar uma língua com igual pronúncia e o mesmo espírito do povo que sorve o figo, a pêra, o damasco e a nêspera?

(Le peuple qui suce la noix d'acajou, la mangue, le cambouca et la jabouticaba, peut-il parler une langue avec la même prononciation et le même esprit que le peuple qui déguste la figue, la poire, l'abricot et la nèfle?) (Je souligne) 65 .

63. Lenneberg est le premier à percevoir cette difficulté de l'hypothèse de Whorf. "One of the greatest traps here is that you get verbal responses in two differenty languages, and this is all you have to compare. There is no extralinguistic check on your experiment. This is not, I think, an insuperable difficulty; we can provide extralinguistic checks on our material." Commentaire de Lenneberg dans la section intitulee "The strategy of Research in the Inter-relations of Language and Other Aspects of Culture" dans Hoijer, Language in Culture, op. cit:; p. 266. Voir également Nick J. Enfield, "On linguocentrism", Pütz et Verspoor, Explorations..., op. cit., p. 125-57.

64. Dans la systématisation proposée par Fishman cela constitue le second niveau de l'hypothèse de Whorf caractérisée par la recherche d'événements comportementaux concomitants.

65. Je ne peux m'empêcher de souligner ici le caractère sexuel (cryptique) de cet exemple. La signification première du terme jabuticaba est un fruit (du jabouticabier) de la famille des myrtacées. Or dans l'argot brésilien des proxénètes "jabuticaba" désigne également une femme noire (et par synecdoque le sexe de celle-ci). Étonnamment, en français, le terme "abricot» désigne également le sexe féminin. Voir Diccionário da língua portuguesa, J. Almeida Costa et A. Sampaio e Melo (ed.), $5^{e}$ édition, Porto, Porto Editora, 1981 et Le grand Robert de la langue française, Montréal/Paris, 1988.

L'embarras que j'ai éprouvé à traduire le verbe sorver, dont la signification première est "humer" (et dont "sucer" et "avaler en aspirant" sont les significations secondaires), se dissipe dès lors que l'on perçoit en filigrane la connotation sexuelle introduite par l'exemple de de Alencar. J'ai traduit ce dernier par le verbe "déguster" afin de souligner l'idée de raffinement (élément de la culture) introduit par de Alencar. On peut tout aussi bien sucer (cunnilingus) la jabuticaba que humer l'abricot. La distance que de Alencar désire instaurer entre la culture brésilienne et la culture portugaise se reflète dans le choix des verbes utilisés pour décrire le comportement respectif de ces peuples lorsque vient le moment de manger un fruit. "Déguster" un fruit, comparativement à "sucer" celui-ci, est une marque de civilité et une preuve de l'influence de bonnes mœurs sur le comportement. Somme toute l'intérêt latent de de Alencar est également de démontrer la différence comportementale entre les Brésiliens et les Portugais : les uns ne refoulent pas leurs instincts et sucent volontiers le sexe de leurs femmes, les autres s'adonnent 
Sous le couvert de différences comportementales (sucer/déguster) introduites par des lexèmes appartenant à des cultures différentes (mais de même langue), de Alencar nous introduit sans le savoir au second niveau de l'hypothèse de Whorf. Ce qu'il convient de comparer ici ce sont des différences comportementales induites par l'adoption d'un vocabulaire idoine appartenant à différentes langues. Le locus classicus d'un tel programme demeure depuis le milieu des années cinquante l'étude du langage des couleurs 66 . L'avantage que présente le langage des couleurs est que celui-ci décrit un continuum physique (objectif), tandis que les mots constituant le lexique d'une langue donnée, dont on juge la codifiabilité arbitraire, expriment des catégories cognitives (subjectives) variant au gré des locuteurs.

Il revient à Éric Lenneberg et Roger Brown d'avoirb7 introduit un test empirique visant à vérifier la corrélation entre la "codabilité" (classification des stimuli chromatiques) sur le comportement. Comparant des sujets anglophones et des membres de la tribu des Zunis (indiens du NouveauMexique) quant à leur capacité à se remémorer certaines couleurs, Brown et Lenneberg découvrirent que les résultats de l'expérience étaient déterminés par le lexique de ces langues respectives. La capacité d'une communauté linguistique à reconnaître un échantillon d'une couleur donnée est facilitée par le fait suivant: plus une langue possède un terme usuel pour désigner une couleur, meilleure sera la réponse obtenue si on demande aux sujets de se rappeler les couleurs observées auparavant. Un individu dont la langue établit une distinction entre les couleurs rouge et orange obtiendra de meilleurs résultats, lorsqu'il s'agit de se remémorer un échantillon rouge, qu'un individu dont la langue donne le même terme pour le rouge et l'orange. On pourrait appliquer cette constatation (la loi de Zipf) au sein d'une même communauté linguistique (relativité intralinguistique). II est plus facile à un individu dont le langage comporte les termes amarante, cinabre, cramoisi, magenta ou zinzolin et à qui on aurait présenté une de ces teintes, de s'en souvenir avec succès qu'à un individu qui posséderait uniquement la couleur rouge.

Whorf dit cependant clairement que l'élément déterminant dans son hypothèse de la relativité linguistique concerne le rôle joué par le régime grammatical d'une langue.

aux plaisirs chamels avec retenue et distance en se contentant de humer «l'abricot".

66. Le poète norvégien Rolf Jacobsen dit d'ailleurs que «les couleurs sont les sœurs cadettes des mots» (Farvene er ordenes små søstre). Poème intitulé "Kobolt» (Cobalt), du recueil Fjerntog (1951), colligé dans Rolf Jacobsen, Alle mine dikt (Tout mes poèmes), Oslo, Gyldendal Norsk Forlag, 1990, $4^{e}$ ed., 1996, p. 84.

67. R.W. Brown et E.H. Lenneberg, "A Study in Language and Cognition", Journal of Abnormal and Social Psychology, 49 (1954), p. 454-62. 
One cannot study the behavioral compulsiveness of such material without suspecting a much more far-reaching compulsion from large-sacle patterning or grammatical categories, such as plurality, gender and similar classifications (animate, inanimate, etc.), tenses, voices, and other verb forms, classifications of the type of "parts of speech", and the matter of whether a given experience is denoted by a unit morpheme, an inflected word, or a syntactical combination 68 .

À titre d'exemple la langue hongroise possède l'avantage par rapport à la majorité des autres langues indo-européennes de pouvoir indiquer un mouvement dans l'espace dans toute sa complexité. Prenons par exemple la phrase suivante :

\section{1) Horváth dépose le livre sur la table.}

Le verbe français "déposer» signifie "poser ce que l'on portait; laisser quelque chose quelque part». Le fait que déposer soit un verbe transitif exige donc de spécifier l'objet subissant l'action puis, dans un second temps, la destination de ce même objet. Dans l'exemple précité la table désigne l'endroit (la destination) sur laquelle l'objet (le livre) est déposé. Pour un Hongrois le verbe "déposer» en tant que tel ne signifie rien car celui-ci n'exprime pas un élément essentiel, soit la «trajectoire complète» de l'objet quittant la main de l'agent jusqu'à la destination finale (la table).

FRANÇAIS

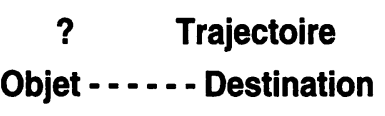

Livre Table

\section{HONGROIS}

\section{Objet—Destination}

Könyv Asztal

En d'autres termes pour qu'un Hongrois comprenne la signification complète du verbe "déposer», celui-ci doit nécessairement introduire une particule décrivant le mouvement dans l'ensemble de sa trajectoire.

\section{2) Horváth felteszi a könyvet az asztalra}

signifie que Horváth dépose le livre sur la table en parcourant un mouvement allant de «bas en haut», tandis que

68. Whorf, "The Relation of Habitual Thought and Behavior to Language", dans Language..., op. cit., p. 137. 


\section{3) Horváth leteszi a könyvet az asztalra}

exprime une trajectoire descendante.

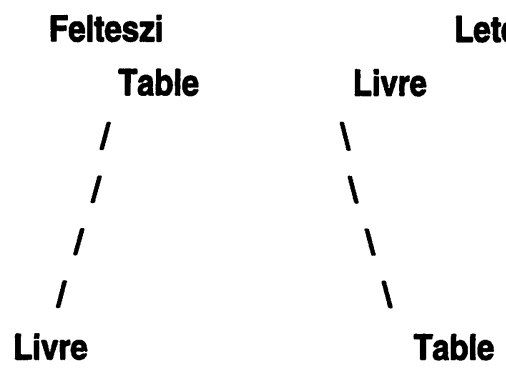

Lorsqu'un Turc rapporte un événement passé celui-ci doit nécessairement indiquer s'il rapporte un événement auquel il a lui-même participé ou bien si c'est un événement rapporté par une tierce personne.

Ils sont arrivés hier soir à Istanbul

se traduit en turc par :

\section{Onlar dün ak_am Istanbuldan geldiler}

si nous avons observé nous-mêmes cet événement. «lls sont arrivés hier soir à Istanbul (et j'étais là pour les accueillir/je les ai vus/etc.)", ou bien par

\section{Onlar dün ak_am Istanbuldan gelmi_ler}

si cet événement nous est rapporté. "lls sont arrivés hier soir à Istanbul (c'est mon frère qui me l'a ditje l'ai lu dans le journal/etc.)

Ces exemples illustrent d'une façon nette les différences conceptuelles (spatiales et temporelles) introduites par différents systèmes grammaticaux. L'hypothèse de la relativité linguistique de Whorf dit en clair que les régimes grammaticaux de différents langages déterminent différemment la catégorisation des concepts généraux (tels que l'espace et le temps).

Nous sommes présentement en mesure de présenter l'hypothèse de la relativité linguistique telle que formulée par Whorf :

Les linguistes découvrent que le système linguistique de base (autrement dit, la grammaire) n'est pas uniquement un instrument reproductif servant à exprimer des idées mais est plutôt le moule «shaper» des idées, le programme et le manuel d'instruction régissant la compréhension de l'activité mentale, l'analyse des impressions, la synthèse des éléments constitutifs essentiels. La formulation des idées ne constitue pas un pro- 
cessus indépendant... mais est lié à une grammaire particulière et ce processus diffère, de peu ou de beaucoup, entre des grammaires différentes. Nous disséquons la nature conformément aux traits dictés par nos langues maternelles 69 .

Cette détermination (ou cette influence) s'exerce-t-elle également sur la philosophie? C'est à cette question que nous allons tenter de répondre dans ce qui suit.

\section{L'hypothèse de Whorf s'applique-t-elle à la philosophie?}

Le philosophe hongrois György Lukács (1885-1917), un intellectuel de génie au demeurant, succomba à la tentation de rédiger ses œuvres philosophiques en allemand. Ce fait - ou cette coquetterie - d'apparence anodine masque toutefois une réalité troublante. Cela correspond chez Lukács au désir effectif de fouler aux pieds la langue hongroise - ravalée au rang d'une langue tout juste utile à servir de lingua franca aux soldats et aux petits fonctionnaires de l'empire austro-hongrois - considérée comme inapte à exprimer des réflexions philosophiques. Le cas Lukács illustre un préjugé bien ancré dans l'esprit de certains individus, voire chez les lettrés en général. Cette idée préconçue reflète le préjugé selon lequel certaines langues seraient plus aptes que d'autres à exprimer des idées philosophiques. À titre d'exemple, les élucubrations "linguistiques" de Heidegger, lequel porta bien haut à un moment de sa vie l'étendard du fascisme le plus obtus, faisaient de l'allemand le véhicule idéal, le vecteur privilégié de la réflexion philosophique ${ }^{70}$. Sur quoi repose une telle supposition? D'aucuns évoquent l'hypothèse de la relativité linguistique afin de justifier un tel parti pris. Cet appel est-il justifiable? C'est à cette question que nous allons tenter de répondre dans ce qui suit.

Nous avons mentionné auparavant que la philosophie, tel un serpent se débarrassant d'une exuvie devenue inutile, a évacué - dès le début des années soixante - l'hypothèse de Whorf. C'est donc avec étonnement que l'on assiste à l'apparition d'une analyse visant à expliquer congrûment la genèse de philosophies nationales influentes (allemande, française, anglaise) par le recours à leur forme grammaticale respective. Le propos n'est pas nécessairement inédit - il s'agit ici de se remémorer les analyses grammaticales de Hans-Georg Koll71. Ce qui est inédit toutefois

69. Traduction libre de l'auteur du texte. Whorf, "Science and Linguistics", dans Language..., op. cit., p. 212-14.

70. Notons, bien entendu, que Heidegger s'inspirait de la thèse de la relativité linguistique de Humboldt et non pas celle de Whorf.

71. Hans-Georg Koll, "»Dynamische" und "statische» Ausdruckstendenzen im Englischen im Vergleich zum Deutschen und Französischen." dans Karl-Richard Bausch et HansMartin Gauger (ed.), Interlinguistica : Sprachvergleich und Übersetzung - Festschrift zum 60. Geburtstag von Mario Wandruszka. Tübingen, Max Niemeyer, 1971, p. 457-82. 
c'est la tentative d'utiliser l'hypothèse de Whorf pour parvenir à cette fin.

Dans un texte fouillé, Wiliam Harvey soutient que la philosophie serait un effet direct de l'adoption d'un système grammatical donné.

"And it is in the comparatively "pure intellectual» domain of philosophy, and in particular of epistemology and ontology, where the fundamentals of world views are straightforwardly elaborated, that the effect of differing patterns of syntax and morphology would seem to be most clearly reflected." (Harvey, op. cit. p. 275)

L'analyse de Harvey porte plus précisément sur les philosophies allemandes, françaises et anglaises. Afin d'illustrer l'influence de la grammaire sur la philosophie, Harvey schématise les différences entre les trois philosophies nationales précitées de la manière suivante :

\begin{tabular}{|l|l|l|l|}
\hline & Mode principal & Principe & Méthode \\
\hline FRANÇAIS & sciemment dualiste & rationaliste & analytique \\
\hline ANGLAIS & sceptique sur la métaphysique & empirique & descriptive \\
\hline ALLEMAND & unitaire & idéaliste & systématique \\
\hline
\end{tabular}

Selon Harvey (s'appuyant lui-même sur les analyses de Hans-Georg Koll) les différences notables entre la philosophie de Descartes et celle de Leibniz se réduiraient essentiellement à une différence entre la grammaire de leur langue respective.

Significantly, the differences in method and emphasis of Leibniz and Descartes correspond closely to the differences in their mother tongues. In German, qualifiers precede nouns and other qualified words, which has the effect of throwing us directly into experience. French, by contrast, qualifies only after the qualified thing has been specified, creating the effect of a chain of methodological analysis.” (Harvey, op. cit., p. 277).

D'une manière analogue, la philosophie de David Hume serait réductible à la tendance marquée de la langue anglaise à utiliser le mode passif : «...Hume's concept...that knowledge is based in experience would seem to be related to the various English passive forms that emphasize the object and connote property rather than activity...»72.

Voilà bien une thèse risible! Si l'ensemble de la production philosophique se réduisait ainsi à une simple question grammaticale, à quel élé-

72. Harvey, op. cit., p. 280. 
ment grammatical fraudrait-il attribuer, par exemple, le caractère génial de l'interprétation atomiste du philosophe Yougoslave (Croate) Rudjer Josip Bo_koviê (1711-1787)? ${ }^{73}$ En d'autres termes quel élément grammatical spécifique au serbo-croate serait déterminant et servirait de pierre angulaire à l'édification de son ontologie? J'ai choisi à dessein l'exemple de Roger Boscovich car ce dernier représente un cas particulièrement intéressant. En effet, l'histoire de la philosophie regorge de contributions provenant d'horizons linguistiques différents de ceux tenus pour prédominants (allemand, français, anglais). Les langues maternelles autres que celles précitées n'ont jamais empêché, par exemple, un philosophe Suédois (Johan Jacob Borelius), Danois (Søren Kierkegaard), Italien (Bertrando Spaventa) ou bien Tchèque (Augustin Smetana) de développer des philosophies idéalistes (de type hégélien) même si celles-ci n'ont absolument rien en commun entre elles (sauf le suédois et le danois) et s'avèrent fondamentalement incompatibles avec le système grammatical allemand. Comment alors expliquer, par exemple, la "capacité" du tchèque à engendrer une philosophie de type idéaliste si cette langue est dépourvue des caractéristiques grammaticales présumées essentielles à l'émergence de ce type de philosophie, soit le régime SOV? ${ }^{74}$ Nous ne sommes pas davantage en mesure d'expliquer la géométrie analytique (antérieure à celle de Descartes d'ailleurs) du Yougoslave Martin GetaldiÊ étant donné que les éléments grammaticaux de sa langue maternelle ne sont pas congruents avec ceux du français. Revenons maintenant à l'exemple de Boscovich. Le croate (ou plus précisément la forme dialectale de l'illyrien parlée à Raguse (aujourd'hui Dubrovnik) était sa langue maternelle, mais c'est en latin et en italien qu'il parfait son éducation et qu'il publie ses œuvres, tant scientifiques, poétiques, que philosophiques. En vertu de la thèse proposée par Harvey, nous sommes dûment autorisés à demander quel élément grammatical serait déterminant dans la formation de la pensée de Boscovich. Serait-ce sa langue maternelle, sa langue de travail (le latin), sa langue de communication (italien) ou bien serait-ce l'influence mutuelle de ces diverses langues (croate, latin, italien) qui auraient servi de creuset à l'élaboration de sa pensée? On sait que Boscovich complète la théorie de Newton en proposant une théorie se situant à mi-chemin entre Newton et Leibniz. Le croate serait-il grammaticalement à mi-chemin entre l'allemand et le français? Le caractère hybride de l'atomisme dynamique de Boscovich tient-il au fait qu'il était

73. Voir principalement Philosophiae naturalis theoria redacta ad unicam legem virium in natura existentium, Vienne, 1758. J'utilise à partir de maintenant la version francisée du nom de l'auteur : Roger Boscovich.

74. Dans les clauses subordonnées en allemand. 
polyglotte? Bien malin celui qui pourrait répondre à ces (pseudo)questions car, force est d'avouer, rien ne permet d'isoler des éléments empiriques servant à étayer de telles suppositions.

Cela étant dit, si la typologie suggérée par Harvey était vraie, il faudrait être en mesure d'expliquer le caractère éminemment "cosmopolite" (dixit Toulmin) de la philosophie, or la thèse de Harvey semble exclure cela. En effet les philosophes - présumés prisonniers de la prison conceptuelle que constituerait leur langue maternelle - sont rarement des individus unilingues. Le Descartes, le Leibniz et le Hume agités par Harvey ne sont pas les philosophes monolingues («linguocentriste!») qu'il désire nous faire "gober"; leur philosophie respective ne jaillit pas inopinément de leur langue maternelle car chez eux le véritable travail conceptuel s'est fait au préalable dans une langue façonnée d'après le moule latin. II ne faut pas oublier, d'ailleurs, que Descartes avait exposé les linéaments de sa philosophie "premièrement en latin", puis en français. Leibniz quant à lui a rédigé une bonne partie de son œuvre philosophique soit en latin ou bien en français. Pour que la thèse suggérée par Harvey soit susceptible d'être vraie, il faudrait, dans un premier temps, dénicher un philosophe complètement "unilingue», ce qui - toutes époques confondues - semble irréaliste. En corollaire, il ne faut pas oublier que la philosophie elle-même s'est bâtie et s'est développée en généralisant la pratique des emprunts interlinguistiques. La philosophie grecque ne s'estelle pas provignée à l'ensemble de la civilisation occidentale par l'entremise du monde arabe?

D'autre part si on adoptait à la lettre la thèse de Harvey, il faudrait expliquer pourquoi les langues présentant des structures grammaticales similaires n'ont pas développé (à un moment ou l'autre de leur histoire) les mêmes systèmes philosophiques. Harvey accorde par exemple beaucoup d'importance au régime grammatical SOV (sujet/objet/verbe) dans l'érection de la philosophie idéaliste. Comment expliquer alors l'absence d'une philosophie comparable à l'idéalisme allemand dans la philosophie turque ou bien japonaise considérant le fait que la forme grammaticale SOV est dominante dans ces deux langues?75 En outre si la thèse de Harvey était vraie il faudrait que tous les philosophes français soient des cartésiens, tous les philosophes allemands des idéalistes et puis tous les philosophes anglais des empiristes. Or l'histoire de la philosophie - que Dieu soit loué!

75. Voir le chapitre intitulé "Dünya bilimleri, dil bilimleri, felsefe" (Sciences du monde, sciences du langage, philosophie) dans Uygur Nermi, Felsefenin ça_risi (Invitation à la philosophie), Istanbul, Remzi Kitabevi, 1984, p. 30-6. En ce qui concerne la philosophie japonaise voir Junko Hamada, Japanische Philosophie nach 1868, Leyde/New York/Cologne, E. J. Brill, 1994. 
- prouve la contraire. Un Pierre Gassendi (empiriste), un Pierre Daniel Huet (1630-1721) sont autant Français que Descartes, pourtant leur philosophie se situe aux antipodes de celle de Descartes. Comment expliquer alors que chez l'un le système grammatical français "détermine» un type de philosophie, alors que chez d'autres "liés au même système grammatical» une toute autre philosophie émerge. Considérant qu'il ne semble pas exister une "détermination" de la grammaire d'une langue sur la constitution d'une philosophie donnée nous sommes justifiés de croire que I'hypothèse de la relativité linguistique de Whorf ne s'applique pas à la philosophie.

\section{Conclusion}

Dans un texte séminal, datant de 1939, Benjamin Lee Whorf prenait soin de bien indiquer les limites de l'hypothèse de la relativité linguistique :

I should be the last to pretend that there is anything so definite as "a correlation" between culture and language and especially between ethnological rubrics such as "agricultural, hunting", etc, and linguistic ones like "infected», "synthetic», or "isolating»" 76 .

Tenant compte de cette mise en garde de Whorf la tentation d'inclure la philosophie parmi les éléments culturels déterminés par le langage s'évanouit. En effet par une lecture attentive de l'hypothèse de la relativité linguistique de Whorf nous rêvons que l'influence qu'exerce le langage sur des éléments constitutifs de la vision du monde (tels que le temps et l'espace) se fait d'une façon inconsciente 77 . Or la philosophie étant une "réflexion consciente et inhabituelle» sur la langue, elle ne saurait être captive d'une détermination unilatérale de la langue sur la culture. C'est d'ailleurs précisément la raison pourquoi Whorf dit que son hypothèse s'applique uniquement aux comportements et aux pensées "habituelles". Cela voudrait dire que les langues naturelles ne déterminent nullement la philosophie (qui est par définition un type de pensée inhabituel). Voilà d'ailleurs pourquoi, à une certaine époque, la philosophie (analytique) a promu l'idée d'un transfert du langage philosophique véhiculé par les langues naturelles (trop susceptibles de créer des faux-problèmes) vers un langage logique (artificiel) universel exempt, en principe, d'ambiguïtés.

S'il est vrai que les différentes langues naturelles influencent notre perception du moment (et je crois que sur cet aspect Whorf a raison) les philosophes en «réfléchissant sur et dans le langage» peuvent se libérer

76. Whorf, "The Relation of Habitual Thought and Behavior to Language", dans Language..., op. cit., p. 138-9.

77. Voir la notion whorfienne de cryptotype dans "Grammatical categories" dans Language..., op. cit., p. 87-101. 
du carcan conceptuel dans lequel elles nous enferment temporairement. II ne faut jamais oublier, après tout, qu'une influence n'est jamais une détermination.

Si grosso modo différentes traditions nationales semblent favoriser à première vue différents types de philosophies, il ne faut pas chercher le fondement de cette disparité dans la langue d'origine mais plutôt dans des facteurs extérieurs comme la tradition, le choix des modèles à imiter et la promotion de styles d'écriture particuliers créant faussement l'impression d'une détermination de la langue sur la philosophie.

\author{
Roch Duval \\ Département de linguistique et de traduction \\ Université de Montréal
}

